\title{
Increase in American Joint Committee on Cancer Stage at Diagnosis for Patients with Skin Cancers after the COVID-19 Lockdown
}

Charlie NARDIN ${ }^{\circledR 1,2}$, LISE SENOT ${ }^{1}$, PAUL PERNOT, Eve PUZENAT ${ }^{1}$, François AUBIN ${ }^{\circledR 1}$ and LUCAS MORIN ${ }^{\circledR 3,4}$

${ }^{1}$ Department of Dermatology, University Hospital, 3 Bd Fleming, FR-25000 Besançon, ${ }^{2}$ UMR 1098 RIGHT, University of Besançon, ${ }^{3}$ Inserm CIC 1431, University Hospital of Besançon, Besançon and ${ }^{4}$ Inserm U1018, High-Dimensional Biostatistics for Drug Safety and Genomics, CESP, Villejuif, France. E-mail: cnardin@chu-besancon.fr

Accepted Nov 29, 2021; Epub ahead of print Nov 29, 2021

During the first surge of the COVID-19 pandemic, the national lockdown (from 17 March to 12 May 2020) in France resulted in a complete reshuffle of the healthcare system. Despite the maintenance of essential health services for urgent diagnostic and therapeutic procedures, strict social distancing measures and uncertainties about the degree of contagiousness of the SARS-CoV-2 virus led patients to avoid seeking hospital and ambulatory care. Previous studies have shown that a substantial proportion of planned outpatient consultations were postponed during this period (1-4). Dermatologists have expressed concern about potential delays in diagnosis of skin cancers, which may lead to suboptimal outcomes in the coming years $(3,5)$. Indeed, it has been reported that the severity of newly diagnosed melanoma was higher after the COVID-19 national lockdown compared with before (6-8). However, published data are limited to melanoma and based mainly on pre-post lockdown comparisons that are potentially affected by seasonal patterns.

To assess whether the severity of newly diagnosed skin cancers increased after the COVID-19 lockdown in France, a retrospective cohort study was conducted using the electronic health records of patients referred to our regional dermatology tumour board (which covers a population of approximately 1.2 million inhabitants). The study included all adult patients newly diagnosed with skin melanoma or cutaneous squamous cell carcinoma between 21 January and 31 August 2020, and divided them into 3 groups according to their date of diagnosis (Fig. 1A): pre-lockdown, lockdown and post-lockdown. Patients diagnosed in 2018 and 2019 during the time interval that mirrors our post-lockdown period (i.e. from 12 May to 31 August) were included to serve as controls. Patients' baseline characteristics were extracted from electronic health records: sex, age at diagnosis, WHO performance status score, histological subtype, Breslow thickness and ulceration status of melanomas, and American Joint Committee on Cancer (AJCC), $8^{\text {th }}$ edition staging. The characteristics of patients are shown in Table I. Their characteristics were similar across the 4 study periods.

The number of patients referred to our regional dermatology tumour board decreased drastically after the national lockdown was enacted, from 73 newly diagnosed patients during the pre-lockdown period to 26 patients during the lockdown mandate. Referrals started increasing again immediately after the lockdown was lifted.
During the post-lockdown period, a tangible worsening of the stages at diagnosis was observed. Hence, 25 of 103 (24\%, 95\% confidence interval (95\% CI): 16-33\%) patients with melanoma were diagnosed at stage III-IV during the post-lockdown period, compared with $7 / 54$ (13\%, 95\% CI 4-22\%) during the pre-lockdown period (absolute difference 11.3\% ( -0.9 to $23.5 \%), p=0.095)$ and 29/201 (14\%, 95\% CI 10-19\%) during the control period in 2018 to 2019 (absolute difference 9.8\% (0.2 to $19.4 \%), p=0.034)$. As shown in Fig. 1B, the risk of
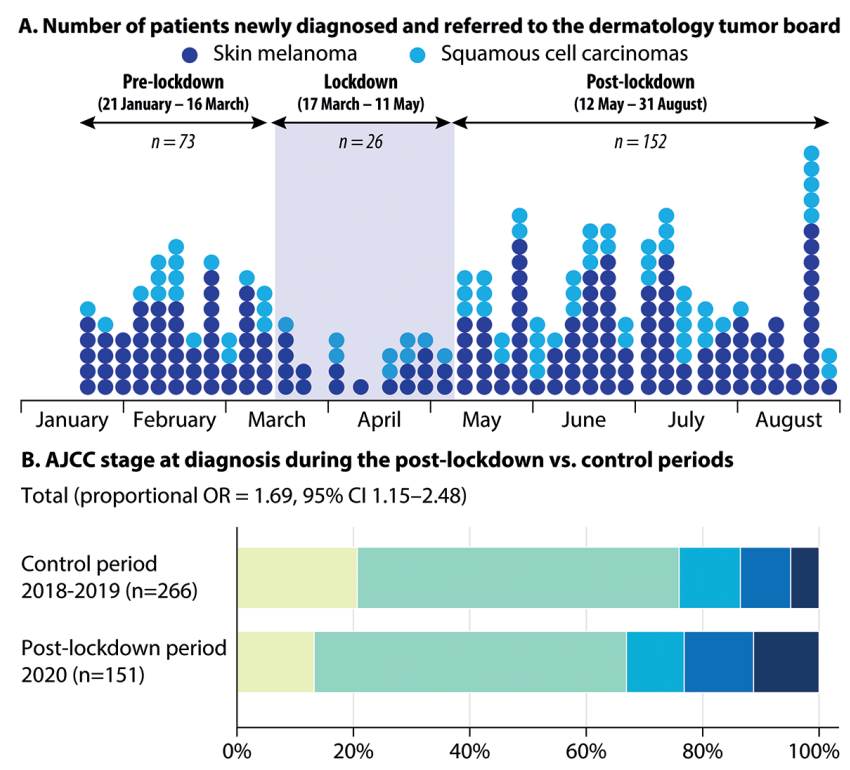

Skin melanoma (proportional OR $=1.65,95 \% \mathrm{Cl} 1.06-2.59$ )

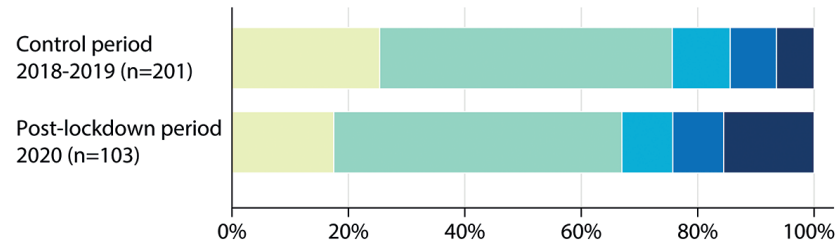

Squamous cell carcinomas (proportional $\mathrm{OR}=1.7395 \% \mathrm{Cl} 0.79-3.78$ )

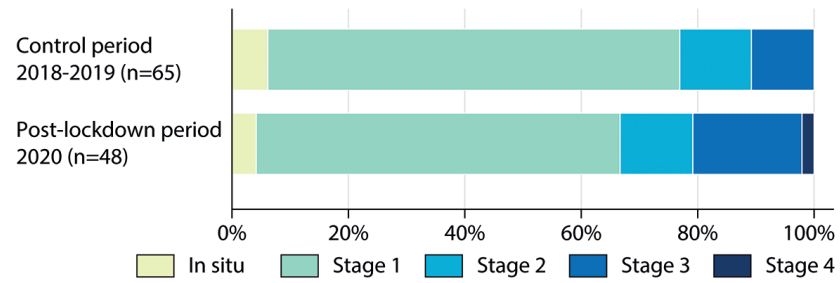

Fig. 1. Skin cancers newly diagnosed during the COVID-19 pandemic. AJCC: American Joint Committee on Cancer; OR: odds ratio; 95\% CI: $95 \%$ confidence interval. 
Table I. Characteristics of patients newly diagnosed with skin melanoma or cutaneous squamous cell carcinoma

\begin{tabular}{|c|c|c|c|c|}
\hline Characteristics & Pre-lockdown ${ }^{a}$ & Lockdown $^{\mathrm{b}}$ & Post-lockdown ${ }^{c}$ & Control period $^{d}$ \\
\hline Patients, $n$ & 73 & 26 & 152 & 269 \\
\hline \multicolumn{5}{|l|}{ Sex, $n(\%)$} \\
\hline Male & $41(56)$ & $16(61)$ & $87(57)$ & $151(56)$ \\
\hline Female & $32(44)$ & $10(39)$ & $65(43)$ & $118(44)$ \\
\hline Age at diagnosis, years, mean (SD) & $66.8(14.7)$ & $75.2(16.1)$ & $70.9(14.8)$ & $69.3(16.5)$ \\
\hline \multicolumn{5}{|l|}{ Skin cancer, $n(\%)$} \\
\hline Melanoma & $55(75)$ & $17(65)$ & $104(68)$ & $204(76)$ \\
\hline Squamous cell carcinoma & $18(25)$ & $9(35)$ & $48(32)$ & $65(24)$ \\
\hline \multicolumn{5}{|l|}{ Performance status, $n(\%)$} \\
\hline 0 & $51(70)$ & $13(50)$ & $104(68)$ & $174(65)$ \\
\hline 1 & $17(23)$ & $9(35)$ & $27(18)$ & $53(20)$ \\
\hline 2 & $2(3)$ & $3(11)$ & $12(8)$ & $29(11)$ \\
\hline 3 & $2(3)$ & $1(4)$ & $7(5)$ & $12(4)$ \\
\hline 4 & $1(1)$ & $0(0)$ & $2(1)$ & $1(0.5)$ \\
\hline Missing stage at diagnosis, $n$ & 1 & 0 & 1 & 3 \\
\hline
\end{tabular}

${ }^{a}$ From 21 January to 16 March 2020. b From 17 March to 11 May 2020. cFrom 12 May to 31 August 2020. dFrom 12 May to 31 August 2018 and 2019.

having a higher AJCC stage at diagnosis increased substantially, with an overall proportional odds ratio of 1.69 (95\% CI 1.15-2.48, $p=0.007)$. In subgroup analyses, results were statistically significant for patients diagnosed with melanoma $(p=0.027)$, but not for patients with squamous cell carcinomas ( $p=0.167)$, owing to the limited sample size. Among the 227 melanoma patients with complete pathological data, mean Breslow depth was $0.90 \mathrm{~mm}$ thicker $(95 \%$ CI $0.25-1.54, p=0.047)$ during the post-lockdown period in 2020 than during the corresponding period in 2018 to 2019 . However, this difference became much more modest and of limited statistical and clinical relevance after excluding 6 individuals with out-of-range Breslow values (mean difference: $0.26 \mathrm{~mm}, 95 \% \mathrm{CI}-0.21$ to $0.73, p=0.190$ ).

The current findings are consistent with previous reports or a sudden and considerable decrease in cancer diagnoses during the first surge of the COVID-19 pandemic (4). In line with other studies, the current study found an increased probability of being diagnosed with more advanced melanoma or squamous cell carcinoma after the lockdown was lifted than before it was implemented. For instance, Ricci et al. (6) reported an increase in Breslow thickness of primary melanoma after the lockdown in Italy. In the USA, Shaikh et al. (7) reported an increase in patients with stage III disease $(30.5 \%$ vs $21.1 \%, p=0.023)$, metastatic recurrence $(7.7 \%$ vs $3.3 \%, p=0.046)$ and brain metastases $(6.5 \%$ vs $1.6 \%$, $p=0.010)$.

Taken together, these results suggest that the drastic measures enforced to contain the spread of the COVID-19 epidemic may have led to potentially harmful delays in the diagnosis of skin cancers. Several limitations should be considered when interpreting the current findings, including the limited sample size, the retrospective nature of data collection, and the regional scope of the study. Future research should evaluate whether the observed worsening of AJCC stages at diagnosis will translate into poorer long-term clinical outcomes.

\section{REFERENCES}

1. Venables ZC, Ahmed S, O Bleiker T, Broggio J, Kwiatkowska $\mathrm{M}$, Levell NJ, et al. The impact of the COVID-19 pandemic on skin cancer incidence and treatment in England, 2020. Br J Dermatol 2021; 185: 460-462.

2. Gomolin T, Cline A, Handler MZ. The danger of neglecting melanoma during the COVID-19 pandemic. J Dermatolog Treat 2020; 31: 444-445.

3. Nardin C, Puzenat E, Dalac S, Maubec E, Aubin F. COVID-19 and skin cancer management: French nation-wide questionnaire survey from real-life practice. J Dermatolog Treat 2020; 24: 1-2.

4. Intergruppo Melanoma Italiano. The effect of COVID-19 emergency in the management of melanoma in Italy. Dermatol Rep 2021; 13: 8972.

5. Blay JY, Boucher S, Le Vu B, Cropet C, Chabaud S, Perol D, et al. Delayed care for patients with newly diagnosed cancer due to COVID-19 and estimated impact on cancer mortality in France. ESMO Open 2021; 6: 100134.

6. Ricci F, Fania L, Paradisi A, Di Lella G, Pallotta S, Sobrino L, et al. Delayed melanoma diagnosis in the COVID-19 era: increased breslow thickness in primary melanomas seen after the COVID-19 lockdown. J Eur Acad Dermatol Venereol 2020; 34: e778-e779.

7. Shaikh S, Yang X, Fortman D, Wang H, Davar D, Luke JJ, et al.Impact of the COVID-19 pandemic on staging at presentation of patients with invasive melanoma. J Clin Oncol 2021; 39: e21579-e21579.

8. Asai Y, Nguyen P, Hanna TP. Impact of the COVID-19 pandemic on skin cancer diagnosis: a population-based study. PLoS One 2021; 16: e0248492. 